NISSUNA UMANA INVESTIGAZIONE SI PUO DIMANDARE VERA SCIENZIA S'ESSA NON PASSA PER LE MATEMATICHE DIMOSTRAZIONI LEONARDO DA VINCI

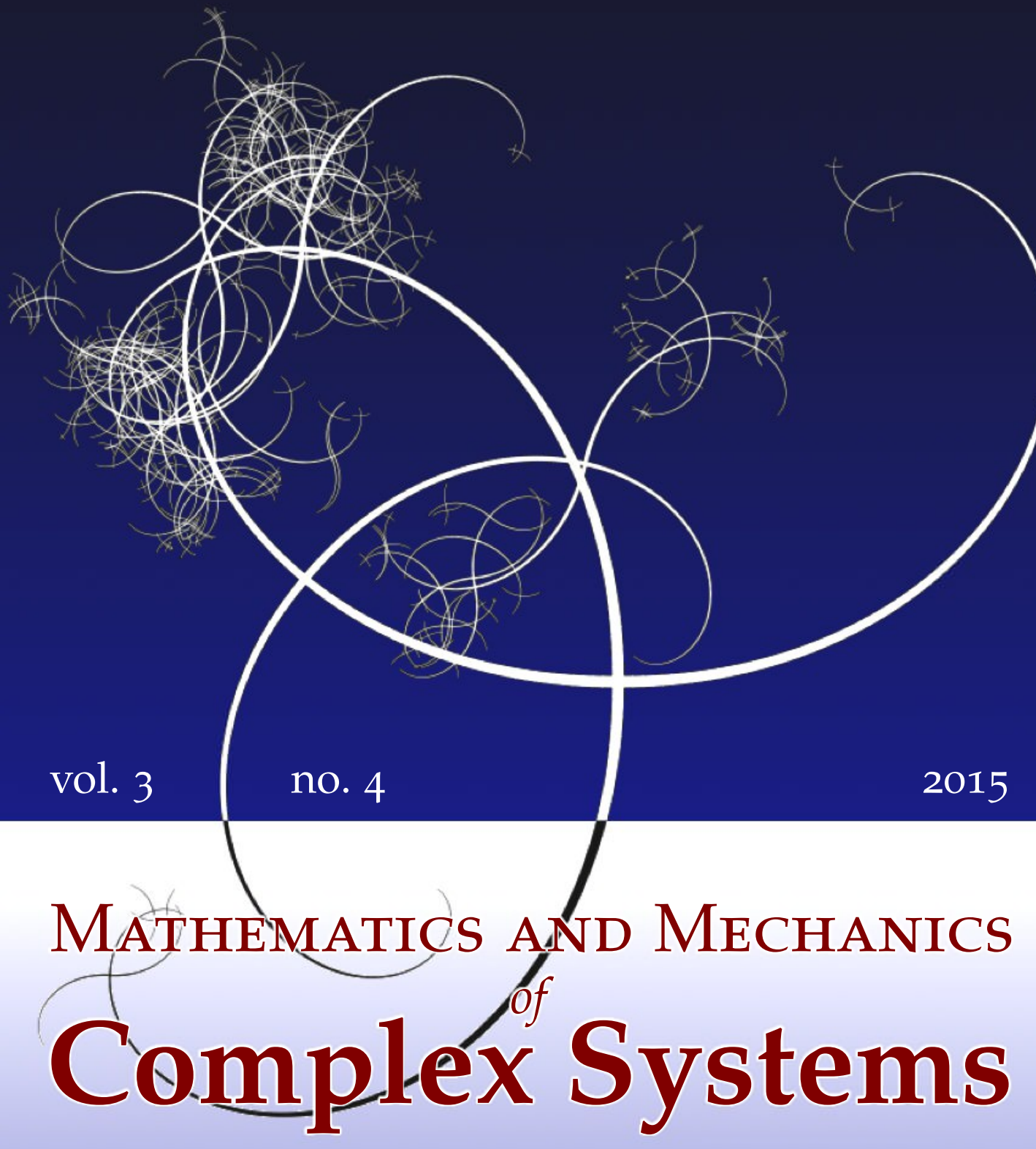

Xu Wang And Peter Schiavone

A CRACK WITH SURFACE ELASTICITY IN FINITE PLANE ELASTOSTATICS 


\title{
A CRACK WITH SURFACE ELASTICITY IN FINITE PLANE ELASTOSTATICS
}

\author{
Xu Wang And Peter Schiavone
}

\begin{abstract}
We consider the effect of surface elasticity on a finite crack in a particular class of compressible hyperelastic materials of harmonic type subjected to uniform remote Piola stresses. The surface mechanics is incorporated into the model of finite deformation by employing a version of the continuum-based surface/interface theory of Gurtin and Murdoch. A complete solution valid throughout the entire domain of interest is obtained by reducing the problem to two series of coupled Cauchy singular integrodifferential equations that are solved numerically using a collocation method. Our model predicts that, in general, the size-dependent Piola stresses exhibit a weak logarithmic singularity at the crack tips. For a crack in a special class of materials subjected to mode II loading, the stresses are bounded whereas the deformation gradients exhibit a logarithmic-type singularity at the crack tips.
\end{abstract}

\section{Introduction}

Analysis of the finite deformation of cracked hyperelastic materials is a challenging topic that, because of its importance, continues to attract the attention of theoreticians and practitioners alike. Knowles and Sternberg [1973; 1974] used asymptotic analysis to study the influence of a crack in compressible hyperelastic homogeneous materials and bimaterials under plane-strain conditions. Knowles [1977] investigated the antiplane shear deformations of a generalized neo-Hookean incompressible material containing a crack. In this investigation, he observed that, in a special class of these materials, the shear stresses at the crack tip are bounded whereas the displacement gradients remain unbounded. Knowles [1981] again used asymptotic analysis to study the influence of a crack in a solid subjected to mode II loading in finite elastostatics. He observed that an antisymmetric solution is impossible and that crack opening at the crack tip still exists under mode II conditions. Also of great interest was the analysis of Knowles and Sternberg [1983], who

\section{Communicated by Antonio Carcaterra.}

MSC2010: 30B99, 45E99, 74B20, 74R99.

Keywords: surface elasticity, hyperelastic material of harmonic type, crack, logarithmic singularity,

Cauchy singular integrodifferential equations. 
studied the crack-tip field of an interface crack in a neo-Hookean bimaterial and found that the classical oscillatory singularities disappear. The asymptotic analysis of a crack in incompressible hyperelastic homogeneous materials and bimaterials was examined further by Geubelle and Knauss [1994a; 1994b; 1994c]. In contrast to the aforementioned asymptotic analyses, $\mathrm{Ru}$ [2002] obtained a complete solution for an interface crack in a bimaterial composed of a particular class of compressible harmonic materials by utilizing a concise version of the complex-variable formulation. Ru's solution indicates that, when the asymptotic behavior of the harmonic materials satisfies the constitutive restriction proposed by Knowles and Sternberg [1975], the oscillatory singularity again disappears.

Most recently, various authors (see, for example, [Kim et al. 2010b; 2010a; 2011a; 2011b; 2011c; Antipov and Schiavone 2011; Wang 2015]) have incorporated the continuum-based surface/interface theory of Gurtin and Murdoch [1975; 1978; Gurtin et al. 1998] into the fracture analysis of linearly elastic solids. It was shown that the incorporation of the Gurtin-Murdoch surface model can suppress the classical strong square-root stress/strain singularity at the crack tip predicted in linear elastic fracture mechanics (LEFM) to the weaker logarithmic singularity [Walton 2012; Kim et al. 2013].

The objective of the present study is to incorporate a version of the GurtinMurdoch surface model into the analysis of the finite plane-strain deformations of a compressible hyperelastic material of harmonic type containing a central crack. The complex-variable method [Ru 2002] is used to reduce the original boundaryvalue problem to two sets of coupled first-order Cauchy singular integrodifferential equations that are solved numerically using Chebyshev polynomials and a collocation method. Furthermore, an elementary closed-form analytic solution is derived for a special material under mode II loading. It is seen from this closed-form solution that all stress components are bounded whereas the deformation gradients exhibit a logarithmic singularity at the crack tips.

\section{Bulk and surface elasticity}

In this study, the bulk material is taken from a particular class of compressible hyperelastic solids of harmonic type whereas the elasticity of the surface is restricted to the class of isotropic linearly elastic materials. This simplifying assumption in the mathematical model is a first step/starting point in the investigation of the contribution of surface elasticity to fracture in this class of nonlinearly elastic materials. In fact, as we detail later, the assumptions of isotropy and linearity in the surface model result in singular integrodifferential equations that are accommodated by existing methods in the literature allowing for relatively easy analysis and solution. In contrast, if the surface-elasticity model is assumed also to be hyperelastic, the 
resulting singular integrodifferential equations become highly nonlinear and are not accommodated by any existing theories of analysis.

Bulk elasticity. In this section, we review the equations governing finite planestrain deformations of a particular class of compressible hyperelastic materials of harmonic type first advanced by John [1960] and later studied by various authors [Ru 2002; Knowles and Sternberg 1975; Varley and Cumberbatch 1980; Li and Steigmann 1993; Wang et al. 2005; Wang and Schiavone 2015]. Let the complex variable $z=x_{1}+i x_{2}$ represent the initial coordinates of a material particle in the undeformed configuration and $w(z)=y_{1}(z)+i y_{2}(z)$ the corresponding spatial coordinates in the deformed configuration. Thus, the displacements $u_{1}$ and $u_{2}$ of a material particle labeled $\left(x_{1}, x_{2}\right)$ are given by $u_{1}=y_{1}-x_{1}$ and $u_{2}=y_{2}-x_{2}$. Define the deformation gradient tensor by the components $(i, j=1,2)$

$$
F_{i j}=\frac{\partial y_{i}}{\partial x_{j}} .
$$

For a particular class of harmonic materials, the strain energy density $W$ defined with respect to the undeformed unit area can be expressed by [Ru 2002; Varley and Cumberbatch 1980; Li and Steigmann 1993; Abeyaratne 1984]

$$
W=2 \mu[F(I)-J], \quad F^{\prime}(I)=\frac{1}{4 \alpha}\left[I+\sqrt{I^{2}-16 \alpha \beta}\right] .
$$

Here $I$ and $J$ are the scalar invariants of the tensor $\boldsymbol{F} \boldsymbol{F}^{T}$ given by

$$
I=\lambda_{1}+\lambda_{2}=\sqrt{F_{i j} F_{i j}+2 J}, \quad J=\lambda_{1} \lambda_{2}=\operatorname{det}\left[F_{i j}\right],
$$

where $\lambda_{1}$ and $\lambda_{2}$ are the principal stretches, $\mu$ is the shear modulus, and $\frac{1}{2} \leq \alpha<1$ and $\beta>0$ are two material constants. A full discussion of the physical implications of both this class of materials and the associated material constants can be found in [Ru 2002]. We note, in particular, one of the well-known limitations of this harmonic material model in that it exhibits unphysical behavior in states of severe compression. Consequently, in what follows, we concern ourselves only with physical problems exhibiting states of strain that are appropriate for this model.

According to the formulation developed by $\mathrm{Ru}$ [2002], the deformation $w$ can be written in terms of two analytic functions $\varphi(z)$ and $\psi(z)$ as

$$
i w(z, \bar{z})=\alpha \varphi(z)+i \overline{\psi(z)}+\frac{\beta z}{\overline{\varphi^{\prime}(z)}},
$$

and the complex Piola stress function $\chi$ is given by

$$
\chi(z, \bar{z})=2 i \mu\left[(\alpha-1) \varphi(z)+i \overline{\psi(z)}+\frac{\beta z}{\overline{\varphi^{\prime}(z)}}\right] .
$$


In addition, the Piola stress components can be written in terms of the Piola stress function $\chi$ as

$$
-\sigma_{21}+i \sigma_{11}=\chi_{, 2}, \quad \sigma_{22}-i \sigma_{12}=\chi_{, 1} .
$$

Surface elasticity. The equilibrium conditions on the surface incorporating interface/surface elasticity can be expressed as [Gurtin and Murdoch 1975; Gurtin et al. 1998; Ru 2010]

$$
\begin{aligned}
{\left[\sigma_{\alpha j} n_{j} \underline{e}_{\alpha}\right]+\sigma_{\alpha \beta, \beta}^{s} \underline{e}_{\alpha} } & =0 & & \text { (tangential direction), } \\
{\left[\sigma_{i j} n_{i} n_{j}\right] } & =\sigma_{\alpha \beta}^{s} \kappa_{\alpha \beta} & & \text { (normal direction), }
\end{aligned}
$$

where $\alpha, \beta=1,3, n_{i}$ is the unit normal vector to the surface before deformation, $[\cdot]$ denotes the jump of the quantities across the surface, $\sigma_{\alpha \beta}^{s}$ is the surface PiolaKirchhoff stress tensor of the first kind, and $\kappa_{\alpha \beta}$ is the curvature tensor of the surface. In addition, the constitutive equations on the isotropic linearly elastic surface are given by

$$
\sigma_{\alpha \beta}^{s}=\sigma_{0} \delta_{\alpha \beta}+2\left(\mu^{s}-\sigma_{0}\right) \varepsilon_{\alpha \beta}^{s}+\left(\lambda^{s}+\sigma_{0}\right) \varepsilon_{\gamma \gamma}^{s} \delta_{\alpha \beta},
$$

where $\varepsilon_{\alpha \beta}^{s}$ is the surface infinitesimal strain tensor, $\delta_{\alpha \beta}$ is the Kronecker delta for the surface, $\sigma_{0}$ is the surface tension, and $\lambda^{s}$ and $\mu^{s}$ are the two surface Lamé parameters. A justification of (7) and (8) can be found in the Appendix.

\section{A crack with surface effects}

We consider the finite plane-strain deformations of a harmonic material weakened by a crack subjected to remote uniform Piola stresses $\left(\sigma_{11}^{\infty}, \sigma_{22}^{\infty}, \sigma_{12}^{\infty}, \sigma_{21}^{\infty}\right)$. The cross section of the crack occupies the segment $[-a, a]$ of the $x_{1}$ axis, and the faces of the crack are assumed to be traction-free, i.e., $\sigma_{12}=\sigma_{22}=0$ on $-a<x_{1}<a$ and $x_{2}= \pm 0$. Let the upper and lower half-planes be designated the " + " and "-" sides of the crack, respectively.

It follows from (7) that the boundary conditions on the crack faces can be specifically written as

$$
\begin{aligned}
\sigma_{11,1}^{s}+\left(\sigma_{12}\right)^{+}-\left(\sigma_{12}\right)^{-} & =0, \\
\left(\sigma_{22}\right)^{+}-\left(\sigma_{22}\right)^{-} & =-\sigma_{0} y_{2,11}^{+} \quad \text { on the upper crack face, } \\
\sigma_{11,1}^{s}+\left(\sigma_{12}\right)^{+}-\left(\sigma_{12}\right)^{-} & =0, \\
\left(\sigma_{22}\right)^{+}-\left(\sigma_{22}\right)^{-} & =-\sigma_{0} y_{2,11}^{-} \quad \text { on the lower crack face, }
\end{aligned}
$$

where $\left(\sigma_{12}\right)^{-}$and $\left(\sigma_{22}\right)^{-}$in $(9 \mathrm{a})$ and $\left(\sigma_{12}\right)^{+}$and $\left(\sigma_{22}\right)^{+}$in (9b) are zero. 
By assuming a coherent interface, the following relationship can then be obtained from (8):

$$
\sigma_{11,1}^{s}=\left(\lambda^{s}+2 \mu^{s}-\sigma_{0}\right) y_{1,11} .
$$

As a result, it follows from (9) and (10) that

$$
\begin{aligned}
& \left(\sigma_{12}\right)^{+}=-\left(\lambda^{s}+2 \mu^{s}-\sigma_{0}\right) y_{1,11}^{+}, \quad \text { on the upper crack face, } \\
& \left(\sigma_{22}\right)^{+}=-\sigma_{0} y_{2,11}^{+} \\
& \left(\sigma_{12}\right)^{-}=\left(\lambda^{s}+2 \mu^{s}-\sigma_{0}\right) y_{1,11}^{-}, \quad \text { on the lower crack face, } \\
& \left(\sigma_{22}\right)^{-}=\sigma_{0} y_{2,11}^{-}
\end{aligned}
$$

which is equivalent to

$$
\begin{aligned}
& \left(\sigma_{12}\right)^{+}+\left(\sigma_{12}\right)^{-}=-\left(\lambda^{s}+2 \mu^{s}-\sigma_{0}\right)\left(y_{1,11}^{+}-y_{1,11}^{-}\right), \\
& \left(\sigma_{12}\right)^{+}-\left(\sigma_{12}\right)^{-}=-\left(\lambda^{s}+2 \mu^{s}-\sigma_{0}\right)\left(y_{1,11}^{+}+y_{1,11}^{-}\right), \\
& \left(\sigma_{22}\right)^{+}+\left(\sigma_{22}\right)^{-}=-\sigma_{0}\left(y_{2,11}^{+}-y_{2,11}^{-}\right), \\
& \left(\sigma_{22}\right)^{+}-\left(\sigma_{22}\right)^{-}=-\sigma_{0}\left(y_{2,11}^{+}+y_{2,11}^{-}\right) .
\end{aligned}
$$

We now define a new analytic function

$$
\theta(z)=-i \psi(z)+\frac{\beta z}{\varphi^{\prime}(z)} .
$$

The deformation $w$ and the complex Piola stress function $\chi$ along the real axis can then be concisely expressed in terms of $\varphi(z)$ and $\theta(z)$ as

$$
\begin{aligned}
i w=\alpha \varphi\left(x_{1}\right)+\overline{\theta\left(x_{1}\right)}, \quad \chi=2 i \mu\left[(\alpha-1) \varphi\left(x_{1}\right)+\overline{\theta\left(x_{1}\right)}\right] & \\
x_{2} & =0,-\infty<x_{1}<+\infty
\end{aligned}
$$

In view of the above expression, $\varphi(z)$ and $\theta(z)$ can be written in the form

$$
\begin{array}{r}
\varphi(z)=\frac{1}{4 \pi \mu} \int_{-a}^{a}\left\{2 \mu\left[b_{1}(\xi)+i b_{2}(\xi)\right]+f_{2}(\xi)-i f_{1}(\xi)\right\} \ln (z-\xi) d \xi+i A z, \text { (16a) } \\
\theta(z)=\frac{1}{4 \pi \mu} \int_{-a}^{a}\left\{2 \mu(\alpha-1)\left[b_{1}(\xi)-i b_{2}(\xi)\right]+\alpha\left[f_{2}(\xi)+i f_{1}(\xi)\right]\right\} \ln (z-\xi) d \xi \\
-i\left(B+\frac{\beta}{A}\right) z, \text { (16b) }
\end{array}
$$

where $b_{1}\left(x_{1}\right), b_{2}\left(x_{1}\right), f_{1}\left(x_{1}\right)$, and $f_{2}\left(x_{1}\right)$ with $-a<x_{1}<a$ are four unknown real functions to be determined and the two complex constants $A$ and $B$ are related to 
the remote uniform Piola stresses through

$$
\begin{aligned}
(1-\alpha) A-\frac{\beta}{\bar{A}} & =\frac{\sigma_{11}^{\infty}+\sigma_{22}^{\infty}+i\left(\sigma_{21}^{\infty}-\sigma_{12}^{\infty}\right)}{4 \mu}, \\
B & =\frac{\sigma_{11}^{\infty}-\sigma_{22}^{\infty}-i\left(\sigma_{12}^{\infty}+\sigma_{21}^{\infty}\right)}{4 \mu} .
\end{aligned}
$$

It is clear that $\varphi(z)$ and $\theta(z)$ given in (16) satisfy the uniform loading condition at infinity. Our task below is to determine the four real functions $b_{1}\left(x_{1}\right), b_{2}\left(x_{1}\right)$, $f_{1}\left(x_{1}\right)$, and $f_{2}\left(x_{1}\right)$ in (16) from the remaining boundary conditions on the crack surfaces. The following limiting values can then be obtained from (16):

$$
\begin{aligned}
\varphi_{+}^{\prime}\left(x_{1}\right)= & \frac{2 \mu\left[b_{2}\left(x_{1}\right)-i b_{1}\left(x_{1}\right)\right]-f_{1}\left(x_{1}\right)-i f_{2}\left(x_{1}\right)}{4 \mu} \\
& +\frac{1}{4 \pi \mu} \int_{-a}^{a} \frac{2 \mu\left[b_{1}(\xi)+i b_{2}(\xi)\right]+f_{2}(\xi)-i f_{1}(\xi)}{x_{1}-\xi} d \xi+i A, \\
\varphi_{-}^{\prime}\left(x_{1}\right)= & \frac{2 \mu\left[-b_{2}\left(x_{1}\right)+i b_{1}\left(x_{1}\right)\right]+f_{1}\left(x_{1}\right)+i f_{2}\left(x_{1}\right)}{4 \mu} \\
& +\frac{1}{4 \pi \mu} \int_{-a}^{a} \frac{2 \mu\left[b_{1}(\xi)+i b_{2}(\xi)\right]+f_{2}(\xi)-i f_{1}(\xi)}{x_{1}-\xi} d \xi+i A, \\
\theta_{+}^{\prime}\left(x_{1}\right)= & \frac{2 \mu(\alpha-1)\left[-b_{2}\left(x_{1}\right)-i b_{1}\left(x_{1}\right)\right]+\alpha\left[f_{1}\left(x_{1}\right)-i f_{2}\left(x_{1}\right)\right]}{4 \mu} \\
& +\frac{1}{4 \pi \mu} \int_{-a}^{a} \frac{2 \mu(\alpha-1)\left[b_{1}(\xi)-i b_{2}(\xi)\right]+\alpha\left[f_{2}(\xi)+i f_{1}(\xi)\right]}{x_{1}-\xi} d \xi \\
& -i\left(B+\frac{\beta}{A}\right), \\
& +\frac{1}{4 \pi \mu} \int_{-a}^{a} \frac{2 \mu(\alpha-1)\left[b_{1}(\xi)-i b_{2}(\xi)\right]+\alpha\left[f_{2}(\xi)+i f_{1}(\xi)\right]}{x_{1}-\xi} d \xi \\
& -i\left(B+\frac{\beta}{A}\right),
\end{aligned}
$$

where $-a<x_{1}<a$; the subscript " + " means the limiting value by approaching the crack from the upper half-plane, and the subscript "-" means the limiting value by approaching the crack from the lower half-plane. 
By imposing the boundary conditions in (13), and making use of (18) and (19) in conjunction with (4)-(6), we obtain the hypersingular integrodifferential equations

$$
\begin{gathered}
-\frac{4 \mu(1-\alpha)}{\pi} \int_{-a}^{a} \frac{b_{1}(\xi)}{\xi-x_{1}} d \xi+\frac{2 \alpha-1}{\pi} \int_{-a}^{a} \frac{f_{2}(\xi)}{\xi-x_{1}} d \xi+2 \sigma_{12}^{\infty} \\
=\left(\lambda^{s}+2 \mu^{s}-\sigma_{0}\right) b_{1}^{\prime}\left(x_{1}\right), \\
f_{2}\left(x_{1}\right)=\frac{\sigma_{0}(2 \alpha-1)}{\pi} \int_{-a}^{a} \frac{b_{1}(\xi)}{\left(\xi-x_{1}\right)^{2}} d \xi+\frac{\sigma_{0} \alpha}{\pi \mu} \int_{-a}^{a} \frac{f_{2}(\xi)}{\left(\xi-x_{1}\right)^{2}} d \xi, \\
-\frac{4 \mu(1-\alpha)}{\pi} \int_{-a}^{a} \frac{b_{2}(\xi)}{\xi-x_{1}} d \xi-\frac{2 \alpha-1}{\pi} \int_{-a}^{a} \frac{f_{1}(\xi)}{\xi-x_{1}} d \xi+2 \sigma_{22}^{\infty}=\sigma_{0} b_{2}^{\prime}\left(x_{1}\right), \\
f_{1}\left(x_{1}\right)=-\frac{(2 \alpha-1)\left(\lambda^{s}+2 \mu^{s}-\sigma_{0}\right)}{\pi} \int_{-a}^{a} \frac{b_{2}(\xi)}{\left(\xi-x_{1}\right)^{2}} d \xi \\
+\frac{\alpha\left(\lambda^{s}+2 \mu^{s}-\sigma_{0}\right)}{\pi \mu} \int_{-a}^{a} \frac{f_{1}(\xi)}{\left(\xi-x_{1}\right)^{2}} d \xi,
\end{gathered}
$$

where $-a<x_{1}<a$.

In addition, the following conditions can be obtained from (18), (19) and (4)-(6):

$$
\begin{aligned}
\Delta y_{1} & =y_{1}^{+}-y_{1}^{-}=-\int_{-a}^{x_{1}} b_{1}(\xi) d \xi, \\
\Delta y_{2} & =y_{2}^{+}-y_{2}^{-}=-\int_{-a}^{x_{1}} b_{2}(\xi) d \xi, \\
\sigma_{12}^{+}-\sigma_{12}^{-} & =-f_{1}\left(x_{1}\right), \quad-a<x_{1}<a . \\
\sigma_{22}^{+}-\sigma_{22}^{-} & =-f_{2}\left(x_{1}\right),
\end{aligned}
$$

Consequently, the single-valuedness of the displacements and balance of force for a contour surrounding the crack surface require that

$$
\begin{aligned}
\int_{-a}^{a} b_{1}(\xi) d \xi & =0, & & \int_{-a}^{a} b_{2}(\xi) d \xi=0 \\
\int_{-a}^{a} f_{1}(\xi) d \xi & =0, & & \int_{-a}^{a} f_{2}(\xi) d \xi=0 .
\end{aligned}
$$

If the end conditions

$$
\begin{array}{ll}
\mu(2 \alpha-1) b_{1}( \pm a)+\alpha f_{2}( \pm a)=0 & \text { when } \sigma_{0} \neq 0, \sigma_{12}^{\infty} \neq 0, \text { and } \sigma_{22}^{\infty}=0, \\
\mu(2 \alpha-1) b_{2}( \pm a)-\alpha f_{1}( \pm a)=0 & \text { when } \sigma_{0} \neq 0, \sigma_{22}^{\infty} \neq 0, \text { and } \sigma_{12}^{\infty}=0
\end{array}
$$


are met, then (20) and (21) can be written as first-order Cauchy singular integrodifferential equations

$$
\begin{gathered}
-\frac{4 \mu(1-\alpha)}{\pi} \int_{-a}^{a} \frac{b_{1}(\xi)}{\xi-x_{1}} d \xi+\frac{2 \alpha-1}{\pi} \int_{-a}^{a} \frac{f_{2}(\xi)}{\xi-x_{1}} d \xi+2 \sigma_{12}^{\infty} \\
=\left(\lambda^{s}+2 \mu^{s}-\sigma_{0}\right) b_{1}^{\prime}\left(x_{1}\right), \\
f_{2}\left(x_{1}\right)=\frac{\sigma_{0}(2 \alpha-1)}{\pi} \int_{-a}^{a} \frac{b_{1}^{\prime}(\xi)}{\xi-x_{1}} d \xi+\frac{\sigma_{0} \alpha}{\pi \mu} \int_{-a}^{a} \frac{f_{2}^{\prime}(\xi)}{\xi-x_{1}} d \xi \\
-\frac{4 \mu(1-\alpha)}{\pi} \int_{-a}^{a} \frac{b_{2}(\xi)}{\xi-x_{1}} d \xi-\frac{2 \alpha-1}{\pi} \int_{-a}^{a} \frac{f_{1}(\xi)}{\xi-x_{1}} d \xi+2 \sigma_{22}^{\infty}=\sigma_{0} b_{2}^{\prime}\left(x_{1}\right), \\
\quad+\frac{\alpha\left(\lambda^{s}+2 \mu^{s}-\sigma_{0}\right)}{\pi \mu} \int_{-a}^{a} \frac{f_{1}^{\prime}(\xi)}{\xi-x_{1}} d \xi
\end{gathered}
$$

where $-a<x_{1}<a$.

It should be pointed out that the resulting singular integrodifferential equations are linear in nature due to the introduction of the new analytic function $\theta(z)$ in (14) and that the end conditions in (24) are consistent with the discussions in [Kim et al. 2013] in which the idea is fully explained.

In what follows, we address three special cases:

Case 1. If we choose $\alpha=\frac{1}{2}$ for the case in which $F^{\prime}(I) / I$ approaches unity as $I$ tends to infinity [Knowles and Sternberg 1975] (whose proposed constitutive equation is satisfied by the asymptotic behavior of the harmonic material in this case), (20) and (21) simplify to

$$
\begin{array}{rlrl}
-\frac{2 \mu}{\pi} \int_{-a}^{a} \frac{b_{1}(\xi)}{\xi-x_{1}} d \xi+2 \sigma_{12}^{\infty} & =\left(\lambda^{s}+2 \mu^{s}-\sigma_{0}\right) b_{1}^{\prime}\left(x_{1}\right), & \\
-\frac{2 \mu}{\pi} \int_{-a}^{a} \frac{b_{2}(\xi)}{\xi-x_{1}} d \xi+2 \sigma_{22}^{\infty} & =\sigma_{0} b_{2}^{\prime}\left(x_{1}\right), & -a<x_{1}<a . \\
f_{1}\left(x_{1}\right)=f_{2}\left(x_{1}\right) & =0,
\end{array}
$$

Case 2. If $\sigma_{0}=0$ (i.e., the residual surface tension is ignored as in [Kim et al. 2011c] since its contribution is usually negligible), (20) and (21) simplify to

$$
\begin{aligned}
-\frac{4 \mu(1-\alpha)}{\pi} \int_{-a}^{a} \frac{b_{1}(\xi)}{\xi-x_{1}} d \xi+2 \sigma_{12}^{\infty} & =\left(\lambda^{s}+2 \mu^{s}\right) b_{1}^{\prime}\left(x_{1}\right), \\
b_{2}\left(x_{1}\right) & =\frac{\sigma_{22}^{\infty}}{2 \mu(1-\alpha)} \frac{x_{1}}{\sqrt{a^{2}-x_{1}^{2}}}, \quad-a<x_{1}<a . \\
f_{1}\left(x_{1}\right)=f_{2}\left(x_{1}\right) & =0,
\end{aligned}
$$


Case 3. If $\lambda^{s}, \mu^{s} \rightarrow \infty,(20)$ and (21) simplify to

$$
\begin{aligned}
-\frac{\mu}{\pi \alpha} \int_{-a}^{a} \frac{b_{2}(\xi)}{\xi-x_{1}} d \xi+2 \sigma_{22}^{\infty} & =\sigma_{0} b_{2}^{\prime}\left(x_{1}\right), \\
f_{1}\left(x_{1}\right) & =\frac{\mu(2 \alpha-1)}{\alpha} b_{2}\left(x_{1}\right), \quad-a<x_{1}<a . \\
b_{1}\left(x_{1}\right)=f_{2}\left(x_{1}\right) & =0,
\end{aligned}
$$

We remark that the resulting Cauchy singular integrodifferential equations in $(27)_{1,2},(28)_{1}$, and $(29)_{1}$ are similar in structure.

\section{Solution to the Cauchy singular integrodifferential equations}

Set $x=x_{1} / a$ and $t=\xi / a$ in (23)-(26). For convenience, and without loss of generality, we write $b_{i}(x)=b_{i}(a x)=b_{i}\left(x_{1}\right)$ and $f_{i}(x)=f_{i}(a x)=f_{i}\left(x_{1}\right), i=1,2$. Consequently, (23)-(26) can be written in the normalized form

$$
\begin{aligned}
\int_{-1}^{1} \frac{-4(1-\alpha) \hat{b}_{1}(t)+(2 \alpha-1) \hat{f}_{2}(t)}{t-x} d t & =\pi S_{1} \hat{b}_{1}^{\prime}(x)-2 \pi \\
\int_{-1}^{1} \frac{S_{2}(2 \alpha-1) \hat{b}_{1}^{\prime}(t)+S_{2} \alpha \hat{f}_{2}^{\prime}(t)}{t-x} d t & =\pi \hat{f}_{2}(x), \quad-1<x<1, \\
\int_{-1}^{1} \hat{b}_{1}(t) d t=\int_{-1}^{1} \hat{f}_{2}(t) d t & =0, \quad(2 \alpha-1) \hat{b}_{1}( \pm 1)+\alpha \hat{f}_{2}( \pm 1)=0, \\
\int_{-1}^{1} \frac{-4(1-\alpha) \hat{b}_{2}(t)+(2 \alpha-1) \hat{f}_{1}(t)}{t-x} d t & =\pi S_{2} \hat{b}_{2}^{\prime}(x)-2 \pi, \\
\int_{-1}^{1} \frac{S_{1}(2 \alpha-1) \hat{b}_{2}^{\prime}(t)+S_{1} \alpha \hat{f}_{1}^{\prime}(t)}{t-x} d t & =\pi \hat{f}_{1}(x), \quad-1<x<1, \\
\int_{-1}^{1} \hat{b}_{2}(t) d t=\int_{-1}^{1} \hat{f}_{1}(t) d t & =0, \quad(2 \alpha-1) \hat{b}_{2}( \pm 1)+\alpha \hat{f}_{1}( \pm 1)=0,
\end{aligned}
$$

where

$$
\begin{array}{lll}
\hat{b}_{1}(x)=\frac{\mu b_{1}(x)}{\sigma_{12}^{\infty}}, & \hat{f}_{1}(x)=-\frac{f_{1}(x)}{\sigma_{22}^{\infty}}, & S_{1}=\frac{\lambda^{s}+2 \mu-\sigma_{0}}{\alpha \mu}, \\
\hat{b}_{2}(x)=\frac{\mu b_{2}(x)}{\sigma_{22}^{\infty}}, & \hat{f}_{2}(x)=\frac{f_{2}(x)}{\sigma_{12}^{\infty}}, & S_{2}=\frac{\sigma_{0}}{\alpha \mu} .
\end{array}
$$

Equations (30) and (31) are identical in structure in the sense that (31) can be obtained by replacing the subscripts 1 and 2 in (30) by 2 and 1, respectively. In the following, we focus on the solution of (30). 
By utilizing the first and second inverse operators [Chakrabarti and George 1994; Chakrabarti and Hamsapriye 1999]

$$
\begin{aligned}
T_{\text {first }}^{-1} \psi(x) & =\frac{\sqrt{1-x^{2}}}{\pi} \int_{-1}^{1} \psi(t) d t-\frac{\sqrt{1-x^{2}}}{\pi^{2}} \int_{-1}^{1} \frac{\psi(t)}{(t-x) \sqrt{1-t^{2}}} d t, \\
T_{\text {second }}^{-1} \psi(x) & =\frac{1}{\pi \sqrt{1-x^{2}}} \int_{-1}^{1} \psi(t) d t-\frac{1}{\pi^{2} \sqrt{1-x^{2}}} \int_{-1}^{1} \frac{\sqrt{1-t^{2}} \psi(t)}{t-x} d t,
\end{aligned}
$$

where $-1<x<1$, in $(30)_{2}$ and $(30)_{1}$, respectively, and making use of the conditions in $(30)_{3}$, we arrive at

$$
\begin{aligned}
\sqrt{1-x^{2}}\left[-4(1-\alpha) \hat{b}_{1}(x)+(2 \alpha-1) \hat{f}_{2}(x)\right] & =-\frac{1}{\pi} \int_{-1}^{1} \frac{\sqrt{1-t^{2}}\left[S_{1} \hat{b}_{1}^{\prime}(t)-2\right]}{t-x} d t, \\
S_{2}(2 \alpha-1) \hat{b}_{1}^{\prime}(x)+S_{2} \alpha \hat{f}_{2}^{\prime}(x) & =-\frac{\sqrt{1-x^{2}}}{\pi} \int_{-1}^{1} \frac{\hat{f}_{2}^{\prime}(t)}{(t-x) \sqrt{1-t^{2}}} d t
\end{aligned}
$$

where $-1<x<1$.

The two unknown functions $\hat{b}_{1}(x)$ and $\hat{f}_{2}(x)$ are approximated as

$$
\hat{b}_{1}(x)=\sum_{m=0}^{N+1} c_{m} T_{m}(x), \quad \hat{f}_{2}=\sum_{m=0}^{N+1} d_{m} T_{m}(x),
$$

where $T_{m}(x)$ represents the $m$-th Chebyshev polynomial of the first kind and $c_{m}$ and $d_{m}, m=0,1,2, \ldots, N+1$ are $2 N+4$ unknown coefficients to be determined using the collocation method.

Substituting (36) into (35), and using the identities

$$
\begin{aligned}
\frac{d T_{m}(x)}{d x} & =m U_{m-1}(x), \\
\int_{-1}^{1} \frac{U_{m}(t) \sqrt{1-t^{2}}}{t-x} d t & =-\pi T_{m+1}(x), \\
\int_{-1}^{1} \frac{T_{m}(t)}{(t-x) \sqrt{1-t^{2}}} d t & =\pi U_{m-1}(x)
\end{aligned}
$$

with $U_{m}(x)$ being the $m$-th Chebyshev polynomial of the second kind, we arrive at

$$
\begin{gathered}
\sum_{m=0}^{N+1} T_{m}(x)\left\{\sqrt{1-x^{2}}\left[4(1-\alpha) c_{m}-(2 \alpha-1) d_{m}\right]+m S_{1} c_{m}\right\}=2 x, \\
\sum_{m=0}^{N+1} U_{m-1}(x)\left\{m\left[S_{2}(2 \alpha-1) c_{m}+S_{2} \alpha d_{m}\right]+\sqrt{1-x^{2}} d_{m}\right\}=0 .
\end{gathered}
$$


If we select the collocation points given by $x=-\cos (i \pi / N)$ for $i=1,2, \ldots, N$, (38) becomes

$$
\begin{aligned}
\begin{aligned}
\sum_{m=0}^{N+1}(-1)^{m} \cos \left(\frac{m i \pi}{N}\right)\{[4(1-\alpha) & \left.\left.\sin \left(\frac{i \pi}{N}\right)+m S_{1}\right] c_{m}-(2 \alpha-1) \sin \left(\frac{i \pi}{N}\right) d_{m}\right\} \\
& =-2 \cos \left(\frac{i \pi}{N}\right), \quad i=1,2, \ldots, N,
\end{aligned} \\
\sum_{m=0}^{N+1}(-1)^{m} \sin \left(\frac{m i \pi}{N}\right)\left\{m S_{2}(2 \alpha-1) c_{m}+\left[m S_{2} \alpha+\sin \left(\frac{i \pi}{N}\right)\right] d_{m}\right\} \\
=0, \quad i=1,2, \ldots, N-1, \\
\sum_{m=0}^{N+1}\left[m^{2}(2 \alpha-1) c_{m}+m^{2} \alpha d_{m}\right]=0 .
\end{aligned}
$$

In addition, the conditions in $(30)_{3}$ become

$$
\begin{aligned}
\sum_{m=0, m \neq 1}^{N+1} \frac{1+(-1)^{m}}{1-m^{2}} c_{m} & =0, & \sum_{m=0, m \neq 1}^{N+1} \frac{1+(-1)^{m}}{1-m^{2}} d_{m} & =0, \\
\sum_{m=0}^{N+1}\left[(2 \alpha-1) c_{m}+\alpha d_{m}\right] & =0, & \sum_{m=0}^{N+1}(-1)^{m}\left[(2 \alpha-1) c_{m}+\alpha d_{m}\right] & =0 .
\end{aligned}
$$

The $2 N+4$ unknowns $c_{m}$ and $d_{m}, m=0,1,2, \ldots, N+1$, can be uniquely determined by solving the $2 N+4$ independent linear algebraic equations in (39) and (40).

\section{The stress field}

The four real functions $b_{1}\left(x_{1}\right), b_{2}\left(x_{1}\right), f_{1}\left(x_{1}\right)$, and $f_{2}\left(x_{1}\right)$ have been determined in the previous section by solving the ensuing Cauchy singular integrodifferential equations (30) and (31) numerically. This means that the two analytic functions $\varphi(z)$ and $\theta(z)$ are known. In view of (14), the other original analytic function $\psi(z)$ can be given by

$$
\psi(z)=i \theta(z)-\frac{i \beta z}{\varphi^{\prime}(z)} .
$$

The Piola stresses can then be determined by using (5) and (6). Since $b_{1}( \pm a)$, $b_{2}( \pm a), f_{1}( \pm a)$, and $f_{2}( \pm a)$ are all finite when $\sigma_{0} \neq 0$, the Piola stresses exhibit a weak logarithmic singularity at the crack tips when $\sigma_{0} \neq 0$. In particular, the two stress components $\sigma_{12}$ and $\sigma_{22}$ are singularly distributed along the real axis outside 
the crack as

$$
\begin{aligned}
& \frac{\sigma_{12}}{\sigma_{12}^{\infty}}=-\frac{2(1-\alpha)}{\pi} \int_{-1}^{1} \frac{\hat{b}_{1}(t)}{t-x} d t+\frac{2 \alpha-1}{2 \pi} \int_{-1}^{1} \frac{\hat{f}_{2}(t)}{t-x} d t+1, \\
& \frac{\sigma_{22}}{\sigma_{22}^{\infty}}=-\frac{2(1-\alpha)}{\pi} \int_{-1}^{1} \frac{\hat{b}_{2}(t)}{t-x} d t+\frac{2 \alpha-1}{2 \pi} \int_{-1}^{1} \frac{\hat{f}_{1}(t)}{t-x} d t+1,
\end{aligned} \quad x \notin[-1,1],
$$

from which we arrive at the following asymptotic behavior near the crack tips:

$$
\begin{aligned}
& \frac{\sigma_{12}}{\sigma_{12}^{\infty}}=-\frac{\hat{b}_{1}(1)}{2 \pi \alpha} \ln (x-1)+O(1), \\
& \frac{\sigma_{22}}{\sigma_{22}^{\infty}}=-\frac{\hat{b}_{2}(1)}{2 \pi \alpha} \ln (x-1)+O(1), \\
& \frac{\sigma_{12}}{\sigma_{12}^{\infty}}=\frac{\hat{b}_{1}(-1)}{2 \pi \alpha} \ln |x+1|+O(1), \\
& \frac{\sigma_{22}}{\sigma_{22}^{\infty}}=\frac{\hat{b}_{2}(-1)}{2 \pi \alpha} \ln |x-1|+O(1),
\end{aligned}
$$

In the above derivation, we have used the last of the conditions in (30) and (31). Thus, the incorporation of the surface elasticity suppresses the classical strong square-root singularity [Knowles and Sternberg 1983; Abeyaratne 1984; Ru 2002] to the weaker logarithmic one. In addition, $\sigma_{12}$ and $\sigma_{22}$ are regular and distributed on the crack faces as

$$
\begin{array}{ll}
\left(\sigma_{12}\right)^{+}=\frac{\sigma_{12}^{\infty} S_{1} \hat{b}_{1}^{\prime}(x)+\sigma_{22}^{\infty} \hat{f}_{1}(x)}{2}, & \left(\sigma_{12}\right)^{-}=\frac{\sigma_{12}^{\infty} S_{1} \hat{b}_{1}^{\prime}(x)-\sigma_{22}^{\infty} \hat{f}_{1}(x)}{2}, \\
\left(\sigma_{22}\right)^{+}=\frac{\sigma_{22}^{\infty} S_{2} \hat{b}_{2}^{\prime}(x)-\sigma_{12}^{\infty} \hat{f}_{2}(x)}{2}, & \left(\sigma_{22}\right)^{-}=\frac{\sigma_{22}^{\infty} S_{2} \hat{b}_{2}^{\prime}(x)+\sigma_{12}^{\infty} \hat{f}_{2}(x)}{2},
\end{array}
$$

where $-1<x<1$.

It is seen from (30) that the functions $\hat{b}_{1}(x), \hat{b}_{2}(x), \hat{f}_{1}(x)$, and $\hat{f}_{2}(x)$ are dependent on the two parameters $S_{1}$ and $S_{2}$, which are controlled by the crack size. Consequently, our model also predicts that the induced Piola stresses depend on the crack size. In fact, this is evident from (42) and (44). It is deduced from (28) that, if $\sigma_{0}=0$, the stresses exhibit both weak logarithmic and strong square-root singularities at the crack tips.

\section{Results and discussions}

We first show in Figure 1 the two functions $\hat{b}_{1}(x)$ and $\hat{f}_{2}(x)$ obtained for the case $S_{1}=2, S_{2}=1$, and $\alpha=0.8$. It is observed that both $\hat{b}_{1}(x)$ and $\hat{f}_{2}(x)$ are finite 


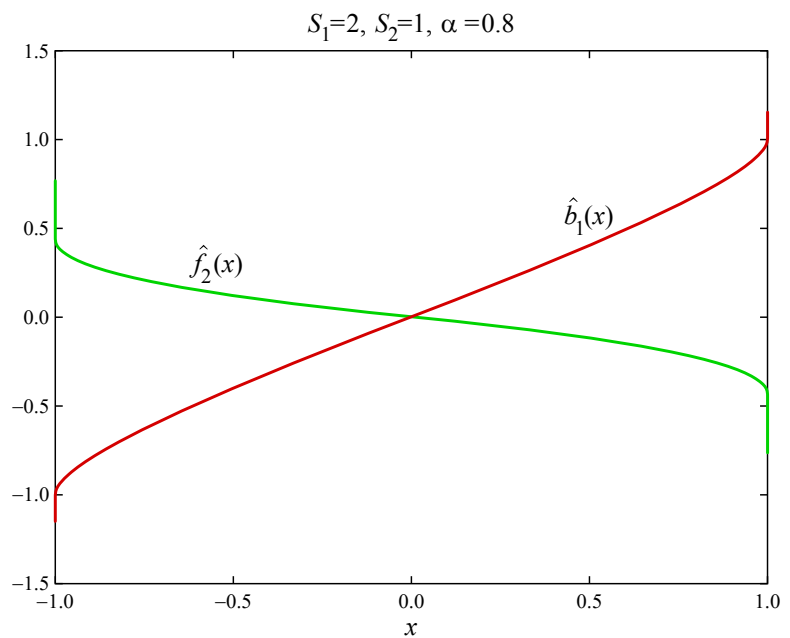

Figure 1. The two functions $\hat{b}_{1}(x)$ and $\hat{f}_{2}(x)$ for $S_{1}=2, S_{2}=1$, and $\alpha=0.8$.

at $x= \pm 1$ (more precisely, $\hat{b}_{1}( \pm 1)= \pm 1.1607$ and $\left.\hat{f}_{2}( \pm 1)=\mp 0.7736\right)$ and that the end conditions $(2 \alpha-1) \hat{b}_{1}( \pm 1)+\alpha \hat{f}_{2}( \pm 1)=0$ are indeed satisfied.

We illustrate in Figure 2 the variations of $\Delta \hat{y}_{1}=\mu \Delta y_{1} /\left(a \sigma_{12}^{\infty}\right)=-\int_{-1}^{x} \hat{b}_{1}(t) d t$ for different values of $\alpha$ with $S_{1}=1$ and $S_{2}=0.1$. We note that, in the presence of surface elasticity, the crack-tip opening angles lie strictly between 0 and $\pi / 2$ and that $\Delta \hat{y}_{1}$ is an increasing function of $\alpha$. We illustrate in Figure $3 \max \left\{\Delta \hat{y}_{1}\right\}$ as functions of $S_{2}$ and $\alpha$ with $S_{1}=1$. From Figure 3, it is clear that $\max \left\{\Delta \hat{y}_{1}\right\}$ lies between the constant value of 0.4958 for $\alpha=\frac{1}{2}$ and the value of 1 for $S_{2}=0$

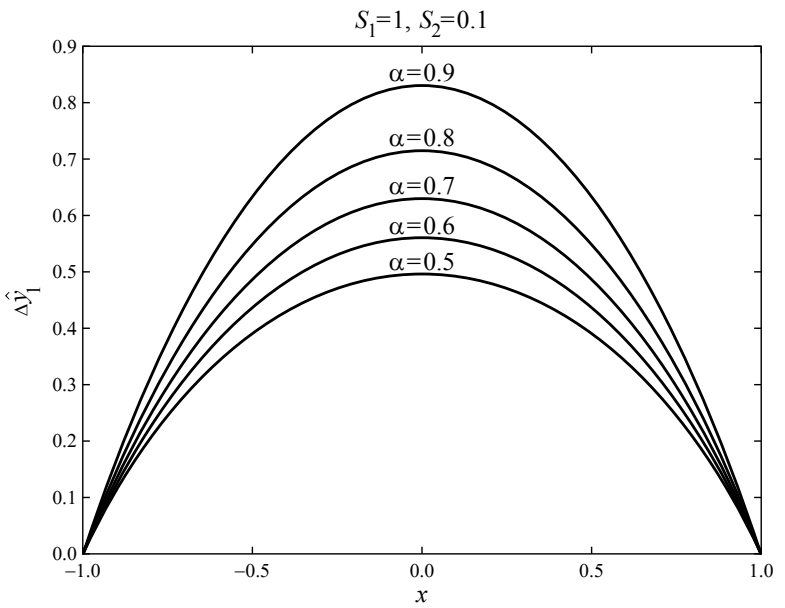

Figure 2. Variations of $\Delta \hat{y}_{1}$ for different values of $\alpha$ with $S_{1}=1$ and $S_{2}=0.1$. 


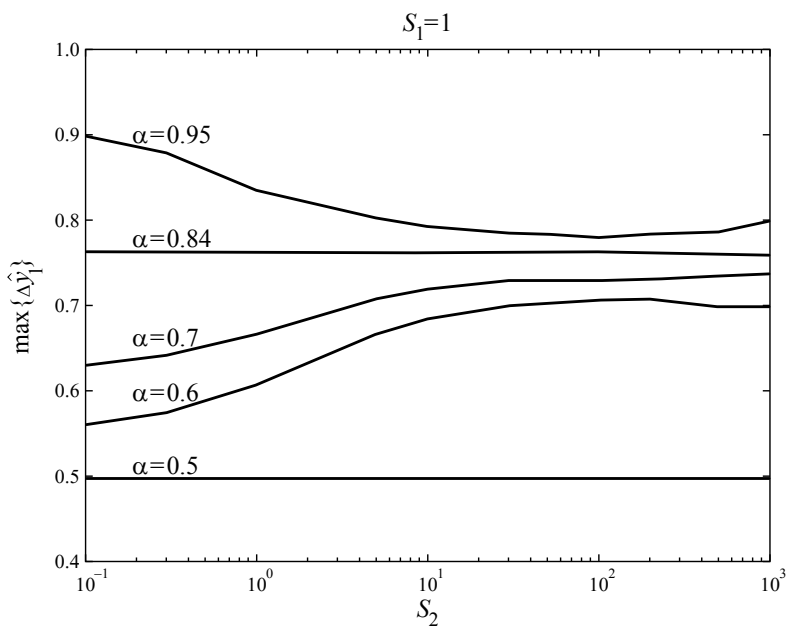

Figure 3. $\max \left\{\Delta \hat{y}_{1}\right\}$ as functions of $S_{2}$ and $\alpha$ with $S_{1}=1$.

and $\alpha=1$, that $\max \left\{\Delta \hat{y}_{1}\right\} \approx 0.76$ when $\alpha=0.84$ for any value of $S_{2}\left(0.1<S_{2}<\right.$ 1000), and that $\max \left\{\Delta \hat{y}_{1}\right\}$ is an increasing function of $\alpha$ but varies in a complicated manner as $S_{2}$ increases. Our numerical results also indicate that $\max \left\{\Delta \hat{y}_{1}\right\} \leq$ $1 / S_{1}$ with equality established when $S_{2}=0$ and $\alpha=1$. It is interesting to note that, when $S_{2}=0$ and $\alpha=1$, we have the exact result: $\Delta \hat{y}_{1}=\left(1-x^{2}\right) / S_{1}$ and $\hat{b}_{1}(x)=2 x / S_{1}$. This fact can be deduced quite easily from (28). In this case, closed-form expressions of the two original analytic functions resulting from the

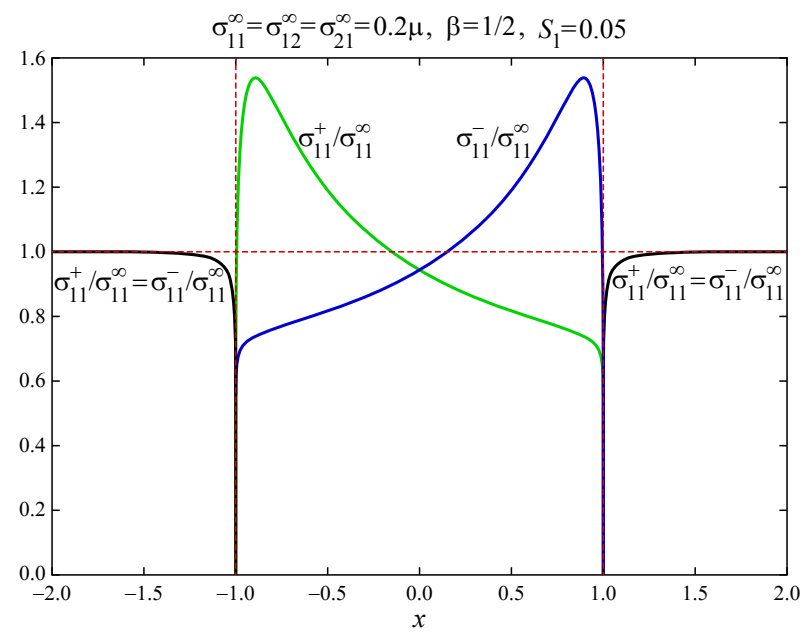

Figure 4. Distribution of the stress component $\sigma_{11}$ along the real axis with $\sigma_{11}^{\infty}=\sigma_{12}^{\infty}=\sigma_{21}^{\infty}=0.2 \mu, \beta=\frac{1}{2}$, and $S_{1}=0.05$. 
mode II loading $\sigma_{11}^{\infty} \neq 0, \sigma_{22}^{\infty}=0$, and $\sigma_{12}^{\infty}=\sigma_{12}^{\infty} \neq 0$ can be explicitly given as

$$
\begin{aligned}
& \varphi^{\prime}(z)=\frac{\sigma_{12}^{\infty}}{\pi \mu S_{1}}\left(-2-\frac{z}{a} \ln \frac{z-a}{z+a}\right)+i A, \\
& \psi(z)=\left(B+\frac{\beta}{A}\right) z-\frac{i \beta z}{\frac{\sigma_{12}^{\infty}}{\pi \mu S_{1}}\left(-2-\frac{z}{a} \ln \frac{z-a}{z+a}\right)+i A},
\end{aligned}
$$

where

$$
A=-\frac{4 \mu \beta}{\sigma_{11}^{\infty}}, \quad B=\frac{\sigma_{11}^{\infty}-2 i \sigma_{12}^{\infty}}{4 \mu}
$$

Evidently, the assumption ensures that the real constant $A$ is finite.

It is further deduced from (14), (15), and (45) that along the $x_{1}$-axis

$$
\begin{array}{ccrl}
\sigma_{12} & =\sigma_{12}^{\infty}, \quad \sigma_{22}=0, & -\infty<x_{1}<+\infty, \\
\sigma_{11}^{+}+i \sigma_{21}^{+}=\frac{\sigma_{11}^{\infty}}{1+\frac{i \sigma_{12}^{\infty} \sigma_{11}^{\infty}}{4 \pi \beta S_{1} \mu^{2}}\left(2+\frac{x_{1}}{a} \ln \left|\frac{x_{1}-a}{x_{1}+a}\right|-\frac{i \pi x_{1}}{a}\right)}+i \sigma_{12}^{\infty}, & \left|x_{1}\right|<a, \\
\sigma_{11}^{-}+i \sigma_{21}^{-}=\frac{\sigma_{11}^{\infty}}{1+\frac{i \sigma_{12}^{\infty} \sigma_{11}^{\infty}}{4 \pi \beta S_{1} \mu^{2}}\left(2+\frac{x_{1}}{a} \ln \left|\frac{x_{1}-a}{x_{1}+a}\right|+\frac{i \pi x_{1}}{a}\right)}+i \sigma_{12}^{\infty}, & \left|x_{1}\right|<a, \\
\sigma_{11}^{+}+i \sigma_{21}^{+}=\sigma_{11}^{-}+i \sigma_{21}^{-} & & \left|x_{1}\right|>a, \\
=\frac{\sigma_{11}^{\infty}}{1+\frac{i \sigma_{12}^{\infty} \sigma_{11}^{\infty}}{4 \pi \beta S_{1} \mu^{2}}\left(2+\frac{x_{1}}{a} \ln \left|\frac{x_{1}-a}{x_{1}+a}\right|\right)}+i \sigma_{12}^{\infty}, & \\
y_{2,1}^{+}=f_{2,1}^{-}=\frac{\sigma_{12}^{\infty}}{\pi \mu S_{1}}\left(2+\frac{x_{1}}{a} \ln \left|\frac{x_{1}-1}{x_{1}+a}\right|\right)+\frac{\sigma_{12}^{\infty}}{2 \mu}, & -\infty<x_{1}<+\infty, \\
y_{1,1}^{+}=-\frac{\sigma_{12}^{\infty}}{a \mu S_{1}} x_{1}-\frac{4 \mu \beta}{\sigma_{11}^{\infty}}, \quad y_{1,1}^{-}=\frac{\sigma_{12}^{\infty}}{a \mu S_{1}} x_{1}-\frac{4 \mu \beta}{\sigma_{11}^{\infty}}, & \left|x_{1}\right|<a, \\
y_{1,1}^{+}=y_{1,1}^{-}=-\frac{4 \mu \beta}{\sigma_{11}^{\infty}}, & \left|x_{1}\right|>a,
\end{array}
$$

which clearly indicates that $y_{2,1}$ exhibits a logarithmic singularity at the crack tips whereas $y_{1,1}$ remains finite at the crack tips.

Figures 4 and 5 show the distributions of $\sigma_{11}$ and $\sigma_{21}$ along the real axis obtained from (47) with $\sigma_{11}^{\infty}=\sigma_{12}^{\infty}=\sigma_{21}^{\infty}=0.2 \mu, \beta=\frac{1}{2}$, and $S_{1}=0.05$. It is seen from Figure 4 that $\sigma_{11}$ is finite and varies continuously along the whole real axis with $\max \left\{\sigma_{11}^{+}\right\}=\max \left\{\sigma_{11}^{-}\right\}=1.5368 \sigma_{11}^{\infty}$ and $\sigma_{11}^{+}=\sigma_{11}^{-}=0$ at the crack tips and that $\sigma_{11} \approx \sigma_{11}^{\infty}$ when $|x|>1.5$. Also, from Figure 5, we see that $\sigma_{21}$ is also finite and again varies continuously along the whole real axis with $\max \left\{\sigma_{21}^{+}\right\}=\max \left\{\sigma_{21}^{-}\right\}=$ $1.8320 \sigma_{21}^{\infty}, \min \left\{\sigma_{21}^{+}\right\}=\min \left\{\sigma_{21}^{-}\right\}=0.7180 \sigma_{21}^{\infty}$, and $\sigma_{21}^{+}=\sigma_{21}^{-}=\sigma_{21}^{\infty}$ at the crack tips 


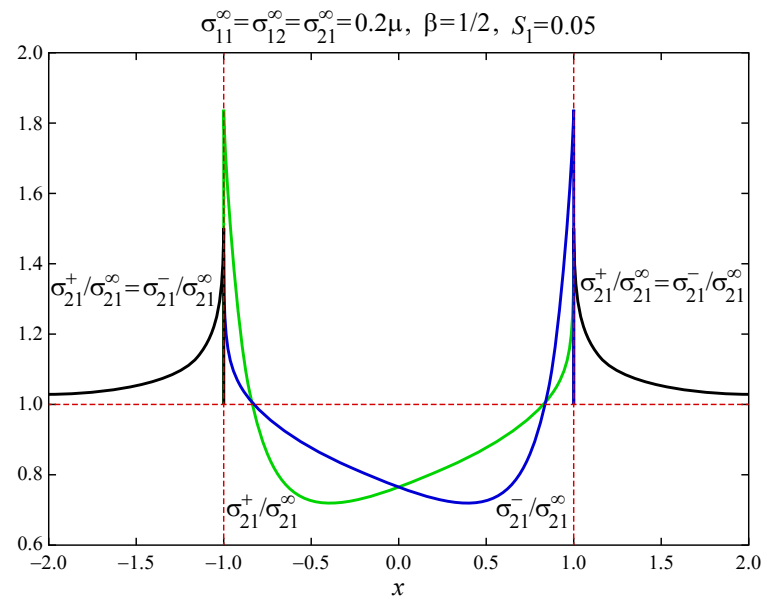

Figure 5. Distribution of the stress component $\sigma_{21}$ along the real axis with $\sigma_{11}^{\infty}=\sigma_{12}^{\infty}=\sigma_{21}^{\infty}=0.2 \mu, \beta=\frac{1}{2}$, and $S_{1}=0.05$.

and at $x= \pm 0.8336$ and that $\sigma_{21}$ outside the crack decays to its remote value much slower than $\sigma_{11}$. It should be stressed that the result $\sigma_{21}^{+}=\sigma_{21}^{-}=\sigma_{21}^{\infty}$ at $x= \pm 0.8336$ is independent of all the loading and material parameters since $x= \pm 0.8336$ are simply the roots of the transcendental equation $2+x(\ln |x-1|-\ln |x+1|)=0$. In this example, we see that all stress components are bounded at the crack tips whereas the deformation gradients exhibit logarithmic singularity at the crack tips.

Figure 6 shows the distributions of $\hat{b}_{1}(x)$ for different values of $S_{1}$ with $\alpha=\frac{1}{2}$. Since $\hat{b}_{1}(x)$ is an odd function of $x$, we demonstrate only the results for $0<x<1$. It is clear that $\hat{b}_{1}(x)$ is finite at $x= \pm 1$ when $S_{1} \neq 0$ and that the magnitude of $\hat{b}_{1}(x)$

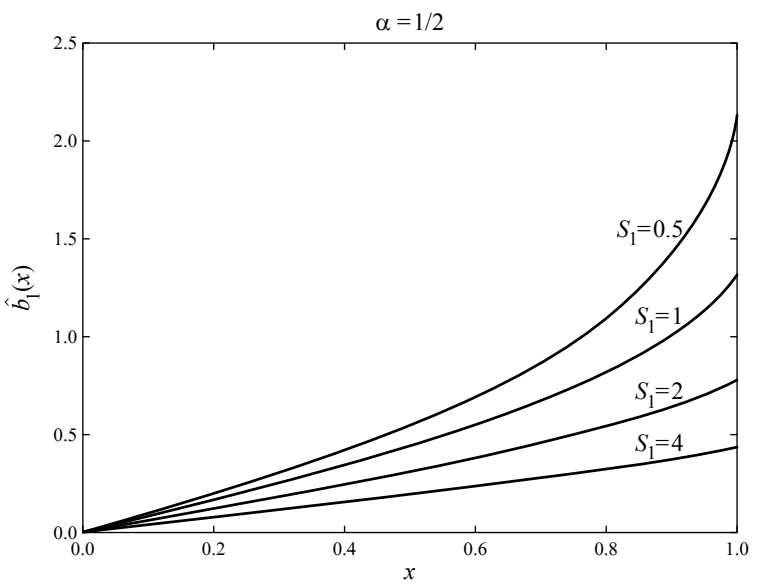

Figure 6. Distributions of $\hat{b}_{1}(x)$ for different values of $S_{1}$ with $\alpha=\frac{1}{2}$. 


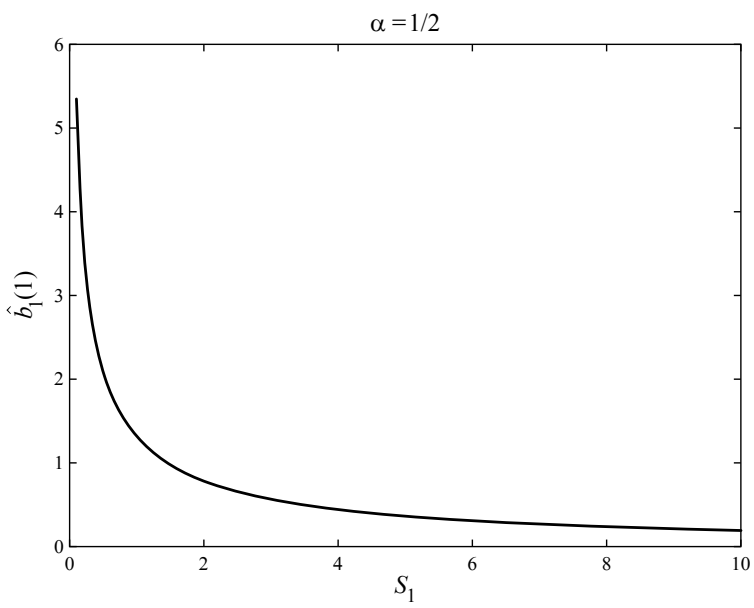

Figure 7. The value $\hat{b}_{1}(1)$ as a function of $S_{1}$ with $\alpha=\frac{1}{2}$.

decreases as $S_{1}$ increases. From (43), we note that $\hat{b}_{1}( \pm 1)$ and $\hat{b}_{2}( \pm 1)$ can be used to characterize the intensity of the logarithmic stress singularity at the crack tips. Figure 7 demonstrates $\hat{b}_{1}(1)$ as a monotonically decreasing function of $S_{1}$ with $\alpha=\frac{1}{2}$. Also, we see that $\hat{b}_{1}(1) \rightarrow \infty$ as $S_{1} \rightarrow 0$ and $\hat{b}_{1}(1) \rightarrow 0$ as $S_{1} \rightarrow \infty$.

\section{Conclusions}

We consider the finite plane-strain deformations of a compressible hyperelastic solid of harmonic type containing a crack whose faces incorporate surface elasticity as described by the Gurtin-Murdoch theory. We obtain a complete solution valid everywhere in the domain of interest (including at the crack tips) by means of two series of coupled Cauchy singular integrodifferential equations (25) and (26). These equations can be simplified considerably for the three cases $\alpha=\frac{1}{2}, \sigma_{0}=0$, and $\lambda^{s}, \mu^{s} \rightarrow \infty$. We propose a method based on Chebyshev polynomials and a collocation technique to solve (25) and (26) numerically. Our results indicate that generally the stresses exhibit a weak logarithmic singularity at the crack tips when the Gurtin-Murdoch model is incorporated. An elementary closed-form solution is obtained in (45) for a material with $\sigma_{0}=0$ and $\alpha=1$ under mode II loading. In this special case, the stresses are found to be bounded at the crack tips.

Finally, we mention that our fundamental hypothesis that the bulk material belongs to a particular class of compressible hyperelastic materials of harmonic type while maintaining the assumption that the crack surfaces are modeled as linearly elastic materials is a first step in analyzing the contribution of the surface in this context. A justification of such a theoretical framework can be found in the Appendix and also in the continuum-based hyperelastic surface elasticity developed by Huang 
and Wang [2006]. We mention also that, if indeed we instead model the crack faces using similar hyperelastic materials, the resulting singular integrodifferential equations become highly nonlinear and are not accommodated by any existing theories in the literature. This makes any further analytical investigations impossible.

\section{Acknowledgements}

We thank two reviewers for their valuable comments and suggestions. This work is supported by the National Natural Science Foundation of China (Grant Number 11272121) and through a Discovery Grant from the Natural Sciences and Engineering Research Council of Canada (Grant Number RGPIN 115112).

\section{Appendix}

Consider a bulk $B \subset \mathbb{R}^{3}$ with surface/interface $\partial B$. The subsurface $S_{C} \subset \partial B$ is enclosed by a simple contour $C$. Let $\boldsymbol{n}$ be the unit normal vector to the subsurface $S_{C}$ before deformation and $\boldsymbol{v}$ be the outward unit normal vector to $C$ before deformation. The force balance condition on the subsurface $S_{C}$ yields

$$
\int_{S_{C}}[\boldsymbol{\sigma} \cdot \boldsymbol{n}]+\int_{C} \boldsymbol{\sigma}^{s} \cdot \boldsymbol{v}=\mathbf{0},
$$

where $\sigma$ is the bulk Piola-Kirchhoff stress tensor of the first kind and $\sigma^{s}$ the surface Piola-Kirchhoff stress tensor of the first kind.

By applying Green's theorem to (49), we obtain

$$
[\boldsymbol{\sigma} \cdot \boldsymbol{n}]+\operatorname{div}_{s} \boldsymbol{\sigma}^{s}=\mathbf{0},
$$

where $\operatorname{div}_{s} \sigma^{s}$ denotes the surface divergence of $\sigma^{s}$. The above can be further written in component forms along the tangential and normal directions of the surface:

$$
\begin{aligned}
& {[\boldsymbol{n} \cdot \boldsymbol{\sigma} \cdot \boldsymbol{n}]=\boldsymbol{\sigma}^{s}: \boldsymbol{\kappa},} \\
& {[\boldsymbol{p} \cdot \boldsymbol{\sigma} \cdot \boldsymbol{n}]=-\operatorname{grad}_{s} \boldsymbol{\sigma}^{s},}
\end{aligned}
$$

where $\boldsymbol{p}=\boldsymbol{I}-\boldsymbol{n} \otimes \boldsymbol{n}$ with $\boldsymbol{I}$ being the three-dimensional identity tensor, $\boldsymbol{\kappa}$ is the curvature tensor of the surface, and $\operatorname{grad}_{s} \sigma^{s}$ is the gradient of $\sigma^{s}$ on the surface before deformation. Equation (51) is equivalent to (7). The balance conditions in (51) or (7) are in fact valid whether the specific constitutive equations of the bulk and the surface are linear or nonlinear and whether the deformations are finite or infinitesimal.

In this study, we adopt a linearized isotropic constitutive equation for the surface. As in [Huang and Wang 2006], if the surface Cauchy stress tensor $\boldsymbol{\tau}^{s}$ is taken as

$$
\boldsymbol{\tau}^{s}=\sigma_{0} \boldsymbol{I}_{2}+\lambda^{s} \operatorname{tr}\left(\boldsymbol{\varepsilon}^{s}\right) \boldsymbol{I}_{2}+2 \mu^{s} \boldsymbol{\varepsilon}^{s},
$$


with $\boldsymbol{I}_{2}$ being the two-dimensional identity tensor, the linearized constitutive relation for the surface Piola-Kirchhoff stress tensor of the first kind can then be written as

$$
\boldsymbol{\sigma}^{s}=\sigma_{0} \boldsymbol{I}_{2}+\left(\lambda^{s}+\sigma_{0}\right) \operatorname{tr}\left(\boldsymbol{\varepsilon}^{s}\right) \boldsymbol{I}_{2}+2\left(\mu^{s}-\sigma_{0}\right) \boldsymbol{\varepsilon}^{s}+\sigma_{0} \operatorname{grad}_{s} \boldsymbol{u} .
$$

If we discard the last term $\sigma_{0} \operatorname{grad}_{s} \boldsymbol{u}$, (53) will reduce to (8).

\section{References}

[Abeyaratne 1984] R. Abeyaratne, "Some finite elasticity problems involving crack-tips", Chapter 1, pp. 3-24 in Modelling problems in crack tip mechanics: proceedings of the tenth Canadian Fracture Conference (Waterloo, Canada, 1983), edited by J. T. Pindera and B. R. Krasnowski, Martinus Nijhoff, Dordrecht, 1984.

[Antipov and Schiavone 2011] Y. A. Antipov and P. Schiavone, "Integro-differential equation for a finite crack in a strip with surface effects", Quart. J. Mech. Appl. Math. 64:1 (2011), 87-106.

[Chakrabarti and George 1994] A. Chakrabarti and A. J. George, "Solution of a singular integral equation involving two intervals arising in the theory of water waves", Appl. Math. Lett. 7:5 (1994), 43-47.

[Chakrabarti and Hamsapriye 1999] A. Chakrabarti and Hamsapriye, "Numerical solution of a singular integro-differential equation”, Z. Angew. Math. Mech. 79:4 (1999), 233-241.

[Geubelle and Knauss 1994a] P. H. Geubelle and W. G. Knauss, "Finite strains at the tip of a crack in a sheet of hyperelastic material, I: Homogeneous case", J. Elasticity 35:1-3 (1994), 61-98.

[Geubelle and Knauss 1994b] P. H. Geubelle and W. G. Knauss, "Finite strains at the tip of a crack in a sheet of hyperelastic material, II: Special bimaterial cases", J. Elasticity 35:1-3 (1994), 99-137.

[Geubelle and Knauss 1994c] P. H. Geubelle and W. G. Knauss, "Finite strains at the tip of a crack in a sheet of hyperelastic material, III: General bimaterial case", J. Elasticity 35:1-3 (1994), 139-174.

[Gurtin and Murdoch 1975] M. E. Gurtin and A. I. Murdoch, "A continuum theory of elastic material surfaces”, Arch. Rational Mech. Anal. 57:4 (1975), 291-323.

[Gurtin and Murdoch 1978] M. E. Gurtin and A. I. Murdoch, "Surface stress in solids", Internat. J. Solids Struct. 14:6 (1978), 431-440.

[Gurtin et al. 1998] M. E. Gurtin, J. Weissmüller, and F. Larché, "A general theory of curved deformable interfaces in solids at equilibrium”, Philos. Mag. A 78:5 (1998), 1093-1109.

[Huang and Wang 2006] Z. P. Huang and J. Wang, "A theory of hyperelasticity of multi-phase media with surface/interface energy effect", Acta Mech. 182:3 (2006), 195-210.

[John 1960] F. John, "Plane strain problems for a perfectly elastic material of harmonic type", Comm. Pure Appl. Math. 13:2 (1960), 239-296.

[Kim et al. 2010a] C. I. Kim, P. Schiavone, and C.-Q. Ru, "Analysis of a mode-III crack in the presence of surface elasticity and a prescribed non-uniform surface traction", Z. Angew. Math. Phys. 61:3 (2010), 555-564.

[Kim et al. 2010b] C. I. Kim, P. Schiavone, and C.-Q. Ru, "The effects of surface elasticity on an elastic solid with mode-III crack: complete solution”, J. Appl. Mech. 77:2 (2010), 021011.

[Kim et al. 2011a] C. I. Kim, P. Schiavone, and C.-Q. Ru, "Analysis of plane-strain crack problems (mode-I \& mode-II) in the presence of surface elasticity", J. Elasticity 104:1-2 (2011), 397-420.

[Kim et al. 2011b] C. I. Kim, P. Schiavone, and C.-Q. Ru, "The effect of surface elasticity on a Mode-III interface crack", Arch. Mech. 63:3 (2011), 267-286. 
[Kim et al. 2011c] C. I. Kim, P. Schiavone, and C.-Q. Ru, "Effect of surface elasticity on an interface crack in plane deformations”, Proc. R. Soc. Lond. Ser. A 467:2136 (2011), 3530-3549.

[Kim et al. 2013] C. I. Kim, C.-Q. Ru, and P. Schiavone, "A clarification of the role of crack-tip conditions in linear elasticity with surface effects", Math. Mech. Solids 18:1 (2013), 59-66.

[Knowles 1977] J. K. Knowles, "The finite anti-plane shear field near the tip of a crack for a class of incompressible elastic solids”, Internat. J. Fracture 13:5 (1977), 611-639.

[Knowles 1981] J. K. Knowles, "A nonlinear effect in mode II crack problems", Eng. Fract. Mech. 15:3-4 (1981), 469-476.

[Knowles and Sternberg 1973] J. K. Knowles and E. Sternberg, "An asymptotic finite-deformation analysis of the elastostatic field near the tip of a crack", J. Elasticity 3:2 (1973), 67-107.

[Knowles and Sternberg 1974] J. K. Knowles and E. Sternberg, "Finite-deformation analysis of the elastostatic field near the tip of a crack: reconsideration and higher-order results", J. Elasticity 4:3 (1974), 201-233.

[Knowles and Sternberg 1975] J. K. Knowles and E. Sternberg, "On the singularity induced by certain mixed boundary conditions in linearized and nonlinear elastostatics", Internat. J. Solids Struct. 11:11 (1975), 1173-1201.

[Knowles and Sternberg 1983] J. K. Knowles and E. Sternberg, "Large deformations near a tip of an interface-crack between two Neo-Hookean sheets", J. Elasticity 13:3 (1983), 257-293.

[Li and Steigmann 1993] X. Li and D. J. Steigmann, "Finite plane twist of an annular membrane", Quart. J. Mech. Appl. Math. 46:4 (1993), 601-625.

[Ru 2002] C. Q. Ru, "On complex-variable formulation for finite plane elastostatics of harmonic materials", Acta Mech. 156:3 (2002), 219-234.

[Ru 2010] C. Q. Ru, "Simple geometrical explanation of Gurtin-Murdoch model of surface elasticity with clarification of its related versions", Sci. China Phys. Mech. Astr. 53:3 (2010), 536-544.

[Varley and Cumberbatch 1980] E. Varley and E. Cumberbatch, "Finite deformations of elastic materials surrounding cylindrical holes", J. Elasticity 10:4 (1980), 341-405.

[Walton 2012] J. R. Walton, "A note on fracture models incorporating surface elasticity", J. Elasticity 109:1 (2012), 95-102.

[Wang 2015] X. Wang, "A mode III arc-shaped crack with surface elasticity”, Z. Angew. Math. Phys. 66:4 (2015), 1987-2000.

[Wang and Schiavone 2015] X. Wang and P. Schiavone, "Harmonic three-phase circular inclusions in finite elasticity", Contin. Mech. Thermodyn. 27:4-5 (2015), 739-747.

[Wang et al. 2005] G. F. Wang, P. Schiavone, and C.-Q. Ru, "Harmonic shapes in finite elasticity under nonuniform loading”, J. Appl. Mech. 72:5 (2005), 691-694.

Received 10 May 2015. Revised 20 Aug 2015. Accepted 19 Oct 2015.

XU WANG: xuwang@ecust.edu.cn

School of Mechanical and Power Engineering, East China University of Science and Technology, 130 Meilong Road, Shanghai 200237, China

Peter Schiavone: p.schiavone@ualberta.ca

Department of Mechanical Engineering, University of Alberta, 4-9 Mechanical Engineering Building, Edmonton, Alberta T6G 2G8, Canada 
EDITORIAL BOARD

ANTONIO CARCATERRA

ERIC A. CARLEN

FRANCESCO DELL'ISOLA

RAFFAELE ESPOSITO

ALBERT FANNJIANG

Gilles A. FranCFORT

Pierangelo MARCATI

JEAN-JACQUES MARIGO

PETER A. MARKOWICH

MARTIN OSTOJA-STARZEWSKI

PIERRE SEPPECHER

DAVID J. STEIGMANN

PAUl STEINMANN

PierRe M. SuQueT

MANAGING EDITORS

MICOL AMAR

CORRADO LATTANZIO

ANGELA MADEO

MARTIN OSTOJA-STARZEWSKI

ADVISORY BOARD

ADNAN AKAY

Holm AltenBaCH

MICOL AMAR

HARM ASKES

TEODOR ATANACKOVIĆ

VICTOR BERDICHEVSKY

GUY BOUCHITTÉ

ANDREA BRAIDES

ROBERTO CAMASSA

MAURO CARFORE

ERIC DARVE

FELIX DARVE

ANNA DE MASI

GianPiEtro DEL Piero

EMMANUELE Di BENEDETTO

BERNOLD FIEDLER

IRENE M. GAMBA

DAVID Y. GAO

SERGEY GAVRILYUK

TIMOTHY J. HEALEY

DOMINIQUE JEULIN

ROGER E. KHAYAT

CORRADO LATTANZIO

ROBERT P. LIPTON

ANGELO LUONGO

ANGELA MADEO

JUAN J. MANFREDI

CARLO MARCHIORO

GÉRARD A. MAUGIN

ROBERTO NATALINI PATRIZIO NEFF

ANDREY PIATNITSKI

ERRICO PRESUTTI

MARIO PULVIRENTI

LUCIO RUSSO

Miguel A. F. SANJUAN

PATRICK SElVADURAI

ALEXANDER P. SEYRANIAN

MIROSLAV ŠILHAVÝ

GUIDO SWEERS

ANTOINETTE TORDESILLAS

LEV TRUSKINOVSKY

JUAN J. L. VELÁZQUEZ VINCENZO VESPRI ANGELO VULPIANI msp.org/memocs

Università di Roma "La Sapienza", Italia

Rutgers University, USA

(CO-CHAIR) Università di Roma "La Sapienza", Italia

(TREASURER) Università dell'Aquila, Italia

University of California at Davis, USA

(CO-CHAIR) Université Paris-Nord, France

Università dell'Aquila, Italy

École Polytechnique, France

DAMTP Cambridge, UK, and University of Vienna, Austria

(CHAIR MANAGING EDITOR) Univ. of Illinois at Urbana-Champaign, USA

Université du Sud Toulon-Var, France

University of California at Berkeley, USA

Universität Erlangen-Nürnberg, Germany

LMA CNRS Marseille, France

Università di Roma "La Sapienza", Italia

Università dell'Aquila, Italy

Université de Lyon-INSA (Institut National des Sciences Appliquées), France

(CHAIR MANAGING EDITOR) Univ. of Illinois at Urbana-Champaign, USA

Carnegie Mellon University, USA, and Bilkent University, Turkey

Otto-von-Guericke-Universität Magdeburg, Germany

Università di Roma "La Sapienza", Italia

University of Sheffield, UK

University of Novi Sad, Serbia

Wayne State University, USA

Université du Sud Toulon-Var, France

Università di Roma Tor Vergata, Italia

University of North Carolina at Chapel Hill, USA

Università di Pavia, Italia

Stanford University, USA

Institut Polytechnique de Grenoble, France

Università dell'Aquila, Italia

Università di Ferrara and International Research Center MEMOCS, Italia

Vanderbilt University, USA

Freie Universität Berlin, Germany

University of Texas at Austin, USA

Federation University and Australian National University, Australia

Université Aix-Marseille, France

Cornell University, USA

École des Mines, France

University of Western Ontario, Canada

Università dell' Aquila, Italy

Louisiana State University, USA

Università dell'Aquila, Italia

Université de Lyon-INSA (Institut National des Sciences Appliquées), France University of Pittsburgh, USA

Università di Roma "La Sapienza”, Italia

Université Paris VI, France

Istituto per le Applicazioni del Calcolo "M. Picone", Italy

Universität Duisburg-Essen, Germany

Narvik University College, Norway, Russia

Università di Roma Tor Vergata, Italy

Università di Roma "La Sapienza”, Italia

Università di Roma “Tor Vergata”, Italia

Universidad Rey Juan Carlos, Madrid, Spain

McGill University, Canada

Moscow State Lomonosov University, Russia

Academy of Sciences of the Czech Republic

Universität zu Köln, Germany

University of Melbourne, Australia

École Polytechnique, France

Bonn University, Germany

Università di Firenze, Italia

Università di Roma La Sapienza, Italia

MEMOCS (ISSN 2325-3444 electronic, 2326-7186 printed) is a journal of the International Research Center for the Mathematics and Mechanics of Complex Systems at the Università dell'Aquila, Italy.

Cover image: "Tangle” by $\odot$ John Horigan; produced using the Context Free program (contextfreeart.org).

PUBLISHED BY

7 mathematical sciences publishers

nonprofit scientific publishing

http://msp.org/

(C) 2015 Mathematical Sciences Publishers 
Mathematics and Mechanics of Complex Systems vol. 3 no. 4

An analysis of the latitudinal data of Eratosthenes and Hipparchus

\section{Christian Marx}

Spatial and material stress tensors in continuum mechanics 341 of growing solid bodies

Jean-François Ganghoffer

A crack with surface elasticity in finite plane elastostatics 365 $\mathrm{Xu}$ Wang and Peter Schiavone

An investigation of the active damping of suspension bridges

André Preumont, Matteo Voltan, Andrea Sangiovanni, Renaud Bastaits, Bilal Mokrani and David Alaluf

MEMOCS is a journal of the International Research Center for the Mathematics and Mechanics of Complex Systems at the Università dell' Aquila, Italy.

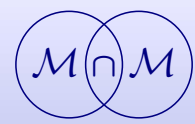

